\title{
DIE WIRTSCHAFT, DURCH STROM GELESEN
}

\section{Elektrizitätssysteme als energiewirtschaftliche Aufschreibesysteme (1880-1930)}

1 Diese Forschung wurde gefördert vom Comité d'histoire de l'électricité et de l'énergie, Fondation Éléctricité de France.

2 Friedrich Kittler: Aufschreibesysteme $1800 / 1900$. Vorwort, in: Zeitschrift für Medienwissenschaft, Bd. 6, Nr. 1, 2011, 117-126.

3 Anson Rabinbach: The Human Motor. Energy, Fatigue, and the Origins of Modernity, New York 1990, 3 f.; M. Norton Wise, Crosbie Smith: Work and Waste: Political Economy and Natural Philosophy in Nineteenth Century Britain (Teil I, II und III), in: History of Science, Bd. 27, Nr. 3, 1989, 263-301, Bd. 27, Nr. 4, 1989, 391-449 und Bd. 28, Nr. 3 , 1990, 221-261.

4 Es ist bezeichnend, dass Stanley Jevons' The Coal Question (1865) von Zeitgenoss_innen als Beitrag zur neoklassischen Ökonomie verstanden wurde (und wohl auch so intendiert war). Erst lange im Nachhinein gilt Jevons als Begründer der Ressourcenökonomie.

\section{Energiewirtschaft schreiben}

Der Text ist ein Versuch, ${ }^{1}$ die Geschichte der Energiewirtschaft nicht als Technikgeschichte, sondern als Geschichte von Aufschreibesystemen zu schreiben, in der das Signifikat <Energiewirtschaft〉 erst entsteht. Unter der Erforschung von Aufschreibesystemen verstehe ich mit Friedrich Kittler die Frage, «was wo in wessen Namen und an welche Adresse zu Papier kommt». ${ }^{2}$ Ich spreche von einem energiewirtschaftlichen Aufschreibesystem, insofern es darum geht, in welcher Sprache, mit welchen Techniken und Medien Energieflüsse in der Gesellschaft aufgezeichnet werden. Energiewirtschaft bezeichnet heute den Bereich technischer und kommerzieller Energie, das heißt die Energie produzierenden, verarbeitenden und konsumierenden Wirtschaftssektoren. Obwohl Energie im I9. Jahrhundert durchaus in massivem Ausmaß technisch hergestellt wurde, blieb diese Erfahrung noch ganz einem <Universum von Arbeitskraft $>$ verpflichtet und verdichtete sich nicht zu einem eigenständigen Bereich technisch hergestellter Energie. Erst zwischen i 880 und I930 werden Elektrizitätssysteme zu einem Aufschreibesystem der Energiewirtschaft: An der Bewegung des Stroms durch die Gesellschaft wird eine Logik technisch hergestellter Energie entwickelt.

Rückblickend erscheint die Energiewirtschaft vor I880 als eine lose Ansammlung von Dampfmaschinen und Kohleöfen, städtischen Gas- und Kerosinlampen sowie weniger Elektromotoren und elektrischer Bogenlampen. Der <energiewirtschaftliche> Zusammenhang dieser Bereiche war noch nicht gegeben. Dem produktivistischen Weltbild dieser Zeit lag es näher, die Dampfkraft ins Verhältnis zur menschlichen Arbeitskraft zu stellen und nicht die Gaswirtschaft. ${ }^{3}$ Es gab keine Position, von der aus die angeeigneten Naturkräfte als dasselbe erscheinen konnten. ${ }^{4}$ Zwar schärfte schon der Übergang zu Kohle bei der Erzeugung von Energie den Blick für die historische Kontingenz und 
die Möglichkeit einer Substitution der Antriebskraft. Sadi Carnot nennt die Dampfmaschine einen «moteur universel», der allmählich Wind- und Wasserräder ersetzen werde, die nur eine lokal beschränkte Aneignung von Naturkräften ermöglichten - eine Beschränkung, mit der die Dampfmaschine breche..$^{5}$ Aber wo diese Vergleiche konkret sind, bleiben sie auf die Antriebskraft beschränkt. Obwohl Ressourcen in der zweiten Hälfte des i 9. Jahrhunderts durchaus mobil waren - der globale Kohlehandel florierte, Gasleitungen durchzogen fortschrittliche Städte und die ersten Ölpipelines wurden gebaut -, sprach man eher von 〈Kraftquellen〉als von <Energieflüssen〉. Die Menschen eigneten sich für ihre Zwecke Arbeitskraft «aus dem allgemeinen Vorrathe der Natur» an, ${ }^{6}$ diese Aneignung blieb jedoch lokal und folgte selbst noch nicht der Logik eines Flusses.

Dagegen geht es in den Elektrizitätssystemen um I930 nicht mehr nur um das Anzapfen von Quellen der Arbeitskraft, sondern um die physikalisch-ökonomische Logik ihrer Verteilung und Nutzung. Im Unterschied zu Kohle, Öl und Gas, die von ihrer Infrastruktur und den Geräten, in denen sie genutzt werden, noch unterscheidbar sind, gibt es das Potenzial elektrischer Arbeit nur als Spannung in einem System. Elektrizität zirkuliert nicht in einer Infrastruktur, sie ist diese Infrastruktur. Gerade weil sie durch und durch technisch ist, ist sie homogen und eindeutig bestimmbar, dabei beweglich und vielfältig umwandelbar: Sie verbindet die Antriebskraft mit der Beleuchtung, magnetische Phänomene mit Wärme. «Der Kreis ist geschlossen», schwärmt Friedrich Engels schon $1883 .{ }^{7}$ In dieser neuen Wirtschaft geht es nicht mehr um den Antrieb einzelner Kraftmaschinen, sondern um einen Fluss künstlicher Arbeit, der die gesamte Gesellschaft durchdringt.

Friedrich Kittlers Aufschreibesysteme 1800/1900 gilt als Intervention, die die technische Vermitteltheit des Schreibens gegen eine Literaturwissenschaft der schreibenden Menschen anführt. ${ }^{8}$ Nicht die Geisteswissenschaft, sondern die Geschichte der Schreibtechniken kann erklären, wer Autor_in sein kann und für wen was geschrieben wird. Der Sinn, den die hermeneutische Geisteswissenschaft verstehen möchte, wird als Begriff überhaupt erst möglich in einer spezifischen Situation technischer Vermittlung. ${ }^{9}$ Kittlers Technikbegriff erschöpft sich jedoch nicht in den Maschinen, Werkzeugen und Geräten, von denen die Technikgeschichte größtenteils spricht. In Anlehnung an Foucaults Arbeiten (und seinen späteren Diskursbegriff) geht es Kittler eher um all das außerbalb einer Aussage, was sie ermöglicht - «the apparatuses of power, storage, transmission, training, reproduction». ${ }^{10}$ Die neuere Techniksoziologie teilt dieses Interesse an «inscription devices»." In Kittlers Arbeit entwickelt sich diese post-hermeneutische Kritik über eine Historiografie der Informationsmedien zu einer mediengeschichtlichen Kritik. ${ }^{12}$ In einer Welt, die durch und durch technisch vermittelt ist, gilt es die Effekte der Medientechniken wie Spuren zu sichern und zu lesen. ${ }^{13}$ Damit wird Mediengeschichte ein Teil der Geschichtsschreibung jedes Gegenstandes - so auch der Energie. ${ }^{14}$
5 Sadi Carnot: Refléxions sur la puissance motrice du feu et sur les machines, Paris 1824, 2; Karl Marx: Das Kapital, Bd. 1, Leipzig 1967 , 398; Andreas Malm: The Origins of Fossil Capital: From Water to Steam in the British Cotton Industry, in: Historical Materialism, Bd. 21, Nr. 1, 2013, 15-68.

6 Hermann von Helmholtz: Über die Wechselwirkung der Naturkräfte und die darauf bezüglichen neuesten Ermittelungen der Physik, o. O., 1854, 22; siehe auch Charles Babbage: On the Economy of Machinery and Manufactures, London 1835, 18.

7 Brief von Friedrich Engels an Eduard Bernstein vom 27.2.1883, in: Karl Marx, Friedrich Engels: MarxEngels-Werke, Bd. 35, Briefe Januar 1881 - März 1883, Berlin 1979, 444 f.

8 Oliver Jahraus: Friedrich Kittler. Paranoia und Theorie, in: Zeitschrift für Medienwissenschaft, Bd. 6, Nr. 1, 2014, 167-171.

9 David E. Wellbery: Foreword, in: Friedrich A. Kittler: Discourse Networks 1800/1900, Stanford 1990, vii-xxxiii, hier xii.

10 Wellbery spricht von einer "presupposition of exteriority" in Kittlers Arbeit, ebd.

11 Bruno Latour: Visualization and Cognition, in: Knowledge and society, Bd. 6, 1986, 1-40.

12 Hans Ulrich Gumbrecht: Nachwort. Mediengeschichte als Wahrheitsereignis. Zur Singularität von Friedrich A. Kittlers Werk, in: Friedrich A. Kittler: Die Wahrheit der technischen Welt, hg. v. Hans Ulrich Gumbrecht, Berlin 2014, 396-422, hier 403; Wellbery: Foreword, xiii.

$13 \mathrm{Vgl}$. Jahraus: Friedrich Kittler, 167; vgl. Kittler: Die Wahrheit der technischen Welt, 119.

14 Wellbery: Foreword, xiii. 
15 Michel Foucault: Die Ordnung der Dinge. Eine Archäologie der Humanwissenschaften, Frankfurt/M. 2009; Daniela Russ: Making sources of energy. The case of coal (1900-1936), in: Innovation. The European Journal of Social Science Research, Bd. 30, Nr. 3, 2017, 1-15; Imre Szeman, Dominic Boyer (Hg.): Energy Humanities. An Anthology, Baltimore 2017.

16 Rudolf Stichweh: Technik, Naturwissenschaft und die Struktur wissenschaftlicher Gemeinschaften. Wissenschaftliche Instrumente und die Entwicklung der Elektrizitätslehre, in: ders.: Wissenschaft, Universität, Profession, Bielefeld 2013, 87-114.

17 Hans-Jörg Rheinberger: Gaston Bachelard and the Notion of ‘Phénomenotechnique, in: Perspectives on Science, Bd. 13, Nr. 3, 2005, 313-328.

18 Crosbie Smith, M. Norton Wise: Energy and Empire. A Biographical Study of Lord Kelvin, Cambridge 1989; Thomas P. Hughes: The Electrification of America. The System Builders, in: Technology and Culture, Bd. 20, Nr. 1, 1979, 124-161; William S. Pretzer: Working at Inventing. Thomas A. Edison and the Menlo Park Experience, Baltimore 2002.

19 Arthur Wilke: Die Elektrizität, ihre Erzeugung und ihre Anwendung in Industrie und Gewerbe, Berlin 1893, 633 .
Die Energiegeschichte ist ein junges Feld, in dem sich Umwelt-, Wirtschaftsund Technikgeschichte im Gegenstand der Energieressourcen treffen. <Energie> markiert dabei wenig mehr als eine Liste von Ressourcen: Es geht eben um Öl, nicht um Baumwolle. Die Wissensordnung, die diese Liste plausibel macht, ist (noch) kein Gegenstand des Feldes. ${ }^{15}$ An einem kleinen Ausschnitt der Energiegeschichte - der Entwicklung von Elektrizitätssystemen zwischen I880 und I930 - möchte ich im Folgenden zeigen, wie Techniken der Dokumentation und Regulierung die technische, wirtschaftliche und politische Aneignung der Elektrizität informiert haben. Dies bedeutet nicht, dass Medientechniken diese Aneignungen determinieren oder ihnen vorhergehen, vielmehr gilt es zu zeigen, inwiefern sie ein Teil von ihnen sind.

\section{Die technische Form der Elektrizität}

Obwohl elektrische Phänomene wie Blitze und geladener Bernstein schon lange bekannt waren, verdichteten sich die unterschiedlichen diesbezüglichen Erfahrungen lange nicht zu einem Phänomen. Erst ihre experimentelle, technische Reproduktion zeigte sie als Phänomene der Auf- und Entladung. Die frühe Elektrizitätslehre war also in besonderem Maße auf die technische Herstellung ihres Phänomens im Labor angewiesen. ${ }^{16}$ Sie kann als eine der ersten «phänomenotechnischen» Wissenschaften verstanden werden, insofern sie natürliche Phänomene nicht nur nach-, sondern herstellte. ${ }^{17}$ Die technische Aneignung von Strom - das heißt des Phänomens fließender anstelle von statischer Ladung oder spontaner Entladung - wurde zuerst experimentell realisiert, bevor man die natürlichen Ströme entdeckte (die als Magnetismus freilich bekannt waren). Anders als in der Mechanik ging die experimentelle Forschung hier schon immer der technischen Nutzung voraus, wobei die Experimentatoren nicht selten zu Unternehmern wurden. ${ }^{18}$

Die technische Form der Elektrizität vermittelt ihre wissenschaftliche und wirtschaftliche Aneignung. Dass in Elektrizität immer schon Form-Arbeit steckt, macht sie homogener, flexibler und universeller als andere Güter: «Während [andere Verbrauchsgüter wie Kohle und Petroleum] [...] durch allerhand lokale und individuelle Bedingungen im Umlaufe und Betriebe behindert werden, wird die Elektrizität eine Allgemeingültigkeit erlangen, wie sie jetzt etwa das Gold besitzt; denn ein gewisses Maß elektrischer Energie ist dasselbe hier und dasselbe in Amerika und Australien..$^{19}$ Die Möglichkeit, elektrischen Strom technisch zu realisieren, lässt jedoch noch vollkommen offen, wie sich damit Geld verdienen lässt. Seit der zweiten Hälfte des I 9. Jahrhunderts wirft die wirtschaftliche Aneignung der Elektrizität andere Fragen der Machbarkeit - insbesondere der Vermarkt- und Finanzierbarkeit - auf, die auf die technische Form der Elektrizität verwiesen bleiben und diese konfigurieren.

Im Zentrum steht dabei der Systemcharakter der Elektrizität. Anders als Gas, Kohle und Öl ist sie nicht von <ihrer> Infrastruktur zu trennen und kann nicht 
gespeichert werden. Ihr physikalischer und technisch-wirtschaftlicher Systemcharakter fallen jedoch nicht zusammen: Physikalisch gesehen handelt es sich bei Strom um in einem geschlossenen Kreislauf zirkulierende Elektronen, womit die Wasser- oder Dampfturbine vor dem Generator streng genommen kein Teil des elektrischen Systems ist. Für die Kontrolle des technisch-wirtschaftlichen Elektrizitätssystems ist die Turbine jedoch zentral - sie bestimmt wesentlich die Kapazität, Effizienz und Rohstoffkosten des Gesamtsystems. Aus einer mediengeschichtlichen Perspektive ist darüber hinaus auch der spezifische Verbrauch der Konsument_innen Teil des Systems, insofern er dokumentiert wird oder das System anderweitig beeinflussen kann. ${ }^{20}$

Elektrizitätssysteme bestehen aus Komponenten, die sich und das Gesamtsystem in jedem Moment gegenseitig beeinflussen. Störungen des Systems können von bloßen Effizienzverlusten bis zu seinem vollständigen Absturz führen. Aus diesem Grund waren Techniken der Schätzung, Kontrolle und Dokumentation des Stroms ein wesentlicher Teil der Konstruktion und des Betriebs elektrischer Systeme. Bis ins 20. Jahrhundert hinein wurde Elektrizität vor allem in industriellen oder städtischen Einzelsystemen produziert, die aus einem Generator bestanden, der Licht oder Antriebskraft für die Nutzung vor Ort lieferte. In diesen stationären Kleinstsystemen waren die Kosten und Risiken des Systems kontrollierbar.

Der Systemcharakter stellte zunächst ein wirtschaftliches Problem, bald jedoch schon eine Möglichkeit dar: Die Forschungen in Menlo Park in den I87oer und I88oer Jahren zeigten, dass auch er wirtschaftlich angeeignet werden konnte. ${ }^{21}$ Das Experimentieren unter wirtschaftlicher Maßgabe rekonfigurierte die technische Form der Elektrizität und akzentuierte noch ihren Systemcharakter. ${ }^{22}$ Ausgangspunkt der Experimente war der Versuch, die starre Systemhaftigkeit der elektrischen Beleuchtungsanlagen zu überwinden, in denen alle Lampen in Reihe geschalten waren und nur gemeinsam brennen konnten. Dies beschränkte die elektrische Beleuchtung auf öffentliche und industrielle Räume, während das einzeln bedienbare Gaslicht auch in städtischen Haushalten brennen konnte. Die Parallelschaltung (Abb. I) war nicht die Erfindung des elektrischen Systems, sondern seine experimentelle Weiterentwicklung entlang der Achse der Systemhaftigkeit: Durch mehr Komponenten, die unabhängiger voneinander waren, entstand eine neue Art der Abhängigkeit zwischen ihnen.
20 Dieses Systemverständnis teilt die Technikgeschichte nicht, vgl. Thomas P. Hughes: Networks of Power. Electrification in Western Society, 1880-1930, Baltimore 1993, 7.

21 Pretzer: Working at Inventing; Hughes: Networks of Power, Kap. 2. 22 Hughes spricht von "systembuilders", siehe Hughes: The Electrification of America. 

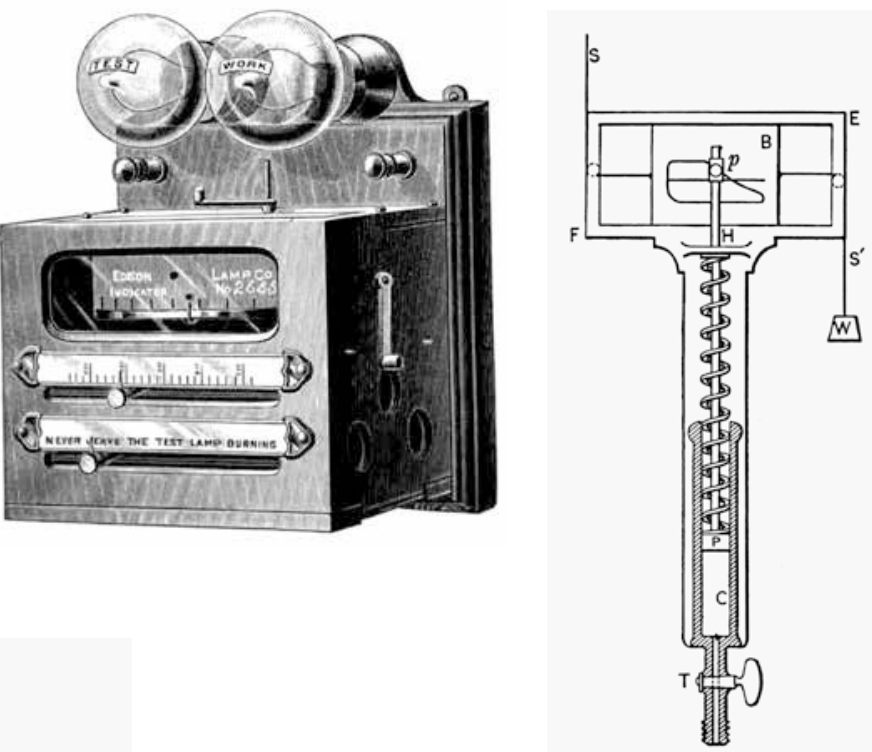
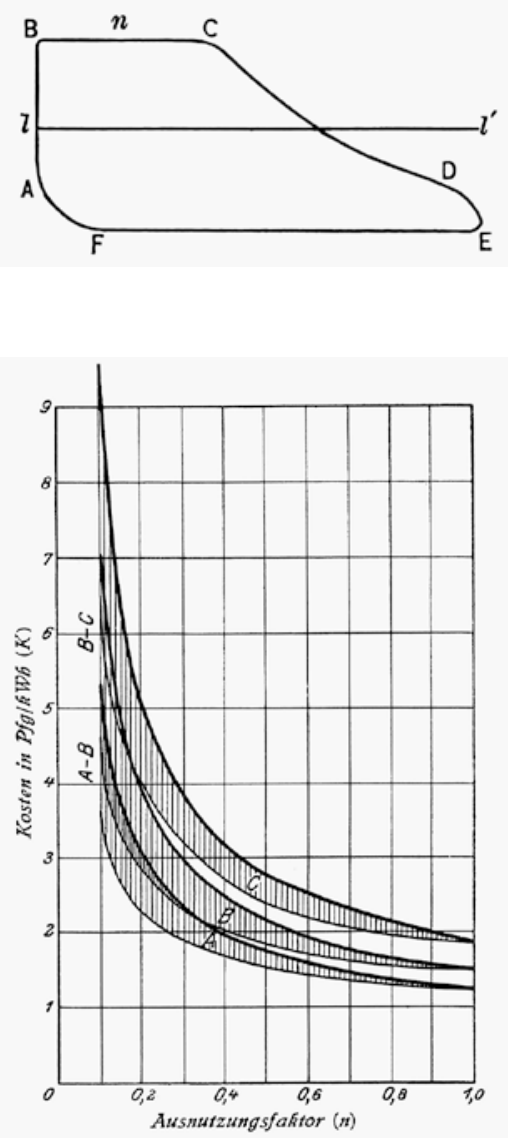

Abb. 18. Abhängigkeit der Erzeugungskosten $K$ von dem Ausnutzungsfaktor $n$ für $f=1$, stark ausgezogene Kurve, für $f=n$, dünner Strich.
Abb. 2-4 v.l.n.r Ein steam indicator (r.) der Druck und Volumen des Dampfkessels im Betrieb als «Carnot'sches Diagramm〉 (1.) aufzeichnet (1900). Mittig Edisons pressure indicator zur Überwachung eines elektrischen Systems, I89I

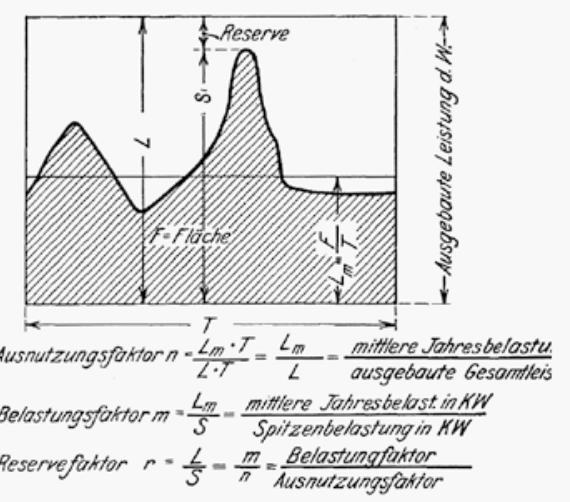

Abb. 5/6 Das Verhältnis von ausgebauter Leistung, Belastung und Kosten in einem Elektrizitätswerk, 1913 


\section{Die Stadt, durch Strom besehen}

Das neue elektrische System war die Zentralstation, die städtische Haushalte und Betriebe über ein Kraftwerk und Verteilernetz zentral versorgen konnte. Obwohl es andere Versuche gab, elektrische Beleuchtungssysteme zu realisieren, galt Edisons System spätestens seit der Internationalen Elektrotechnischen Ausstellung in Paris im Jahr I88I als führend. ${ }^{23}$ In den durchkalkulierten Zentralstationen waren die Verbraucher_innen die unsicherste Komponente. Anders als bei industriellen Kraftwerken hatte der Betreiber keine direkte Kontrolle darüber, wann und wie lange die Lampen brannten. Die Spannung im System musste rund um die Uhr überwacht und gegebenenfalls reguliert werden. Ähnlich wie der steam indicator den Maschineningenieur über den Zustand des Dampfkessels informierte, in den er nicht schauen konnte, vergegenwärtigte der voltage level indicator dem Elektroingenieur den Zustand eines elektrischen Systems, das schon in den I88oer Jahren mehrere Tausend Lampen über Hunderte von Metern umfasste (Abb. 2-4). ${ }^{24}$ Darüber hinaus kannte man den vergangenen Verbrauch über die Stromzähler, die anfangs einfach die Zeit maßen, über die hinweg die Leistung bezogen wurde. Dies alles löste jedoch nicht das tiefergehende wirtschaftliche Problem: Wie kann sichergestellt werden, dass der Verbrauch die Kapazität nicht übersteigt, ohne dass zu viel Kapital ins Vorhalten einer Kapazität gesteckt wird, die so gut wie nie erreicht wird?

Der Betrieb von Zentralstationen lehrte den Zusammenhang der Auslastung des Systems und der Produktionskosten um den Preis des geschäftlichen Ruins. Viele Anlagen scheiterten aus finanziellen Gründen, weil unklar war, wie die Kapitalkosten kalkuliert und eingepreist werden sollten. ${ }^{25}$ Wie bei anderen Versorgungsunternehmen stand das Kapital, das in den Aufbau der Kapazität und des Netzes gesteckt werden musste, in einem Missverhältnis zu den sonstigen Betriebskosten: «By far the most serious problem of central-station management, and by far the greatest item of cost of the product, is interest on investment. $>^{26}$ Die Lösung war Belastungsmanagement - die systematische Entwicklung des Systems hin zu einer stärkeren Auslastung des investierten Kapitals. Im frühen 20. Jahrhundert entstanden zahlreiche Handbücher, die Entwurf, Planung und Betrieb von Zentralstationen unter diese Perspektive stellten. ${ }^{27}$

Die Auslastung einer elektrischen Anlage bezeichnet ihre Ausnutzung im Verhältnis zu ihrer installierten Leistung. Die Berechnungen variieren, doch die Idee bleibt dieselbe: Es ist ein Maß für das brachliegende Kapital. ${ }^{28}$ Zeichnet man dieses Maß über die Zeit auf, erhält man eine Belastungskurve (Abb. 5/6). In einem Handbuch aus dem Jahr I9 3 heißt es dazu knapp: «Einsichtige Betriebsleiter haben längst erkannt, daß durch Umgestaltung der Kurve große wirtschaftliche Vorteile zu erlangen sind, und haben demgemäß durch geschickte Tarifbildung ständig auf ihre Verflachung hingearbeitet [...].«29 Tatsächlich experimentierten Zentralstationen überall mit Tarifen, die nach Höhe und Zeitpunkt des Verbrauchs differenzierten. Stromzähler wurden dabei zu
23 Hughes: Networks of Power, 51. 24 Ebd., $42 \mathrm{f}$.

25 Müllendorf: Ueber die Beurtheilung der Rentabilität elektrischer Anlagen, in: Polytechnisches Journal, Bd. 292, 1894, 110-112; Yakubovich u. a. weisen darauf hin, dass eine Bepreisung nach den Grenzkosten, wie es die Grenznutzentheorie vorgab, in der Elektrizitätswirtschaft nicht möglich war: Die Produktionskosten jeder weiteren Einheit waren billiger, nicht teurer. Siehe Valery Yakubovich, Mark Granovetter, Patrick Mcguire: Electric Charges. The Social Construction of Rate Systems, in: Theory and Society, Bd. 34, Nr. 5/6, 2005, 579-612.

26 Samuel Insull, William Eugene Keily: Central-Station Electric Service. Its Commercial Development and Economic Significance as Set Forth in the Public Addresses (1897-1914) of Samuel Insull, Chicago 1915, 25.

27 Charles H. Merz, William McLellan: Power Station Design, o. O, o. J.; G. Klingenberg: Bau großer Elektrizitätskraftwerke. Erster Band: Richtlinien für den Bau großer Elektrizitätswerke, Berlin 1913; Insull, Keily: Central-Station Electric Service.

$\mathbf{2 8}$ Vgl. Hughes: Networks of Power, $218 \mathrm{f}$.

29 Klingenberg: Bau großer Elektrizitätskraftwerke, 11. 

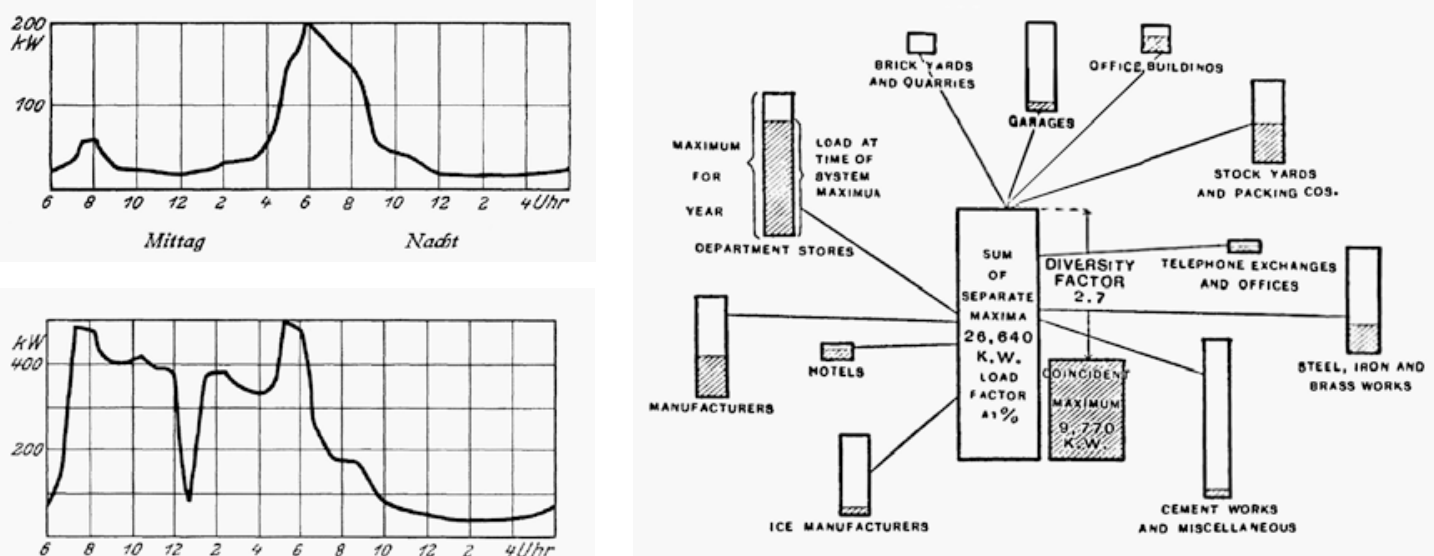

Abb. 7/8 Typische Verbrauchskurven einer Kleinstadt ohne (1.o.) und mit (1.u.) Industrieanschluss, I913

Abb. 9 Die Belastungsverteilung unterschiedlicher städtischer Verbraucher_innen und der Diversitätsfaktor in einem Teil Chicagos, 1915

30 Rheinberger: Gaston Bachelard and the Notion of «Phénomenotechniques, 320 .

31 Insull, Keily: Central-Station Electric Service, 453.

32 William J. Hausman, Peter Hertner, Mira Wilkins: Global Electrification. Multinational Enterprise and International Finance in the History of Light and Power, 1878-2007, Cambridge 2008, 127-8; Jonathan Coopersmith: When Worlds Collide. Government and Electrification, 1892-1939, in: Business and Economic History On-Line, Bd. 1, 2003, 1-31, www.thebhc.org/publications/BEHon line/2003/Coopersmith.pdf(22.6.2020). einem «reified theorem», ${ }^{30}$ in ihnen materialisierte sich das Wissen über einen Zusammenhang von Tarif und Verbrauch, das ihre eigenen Messergebnisse wiederum veränderten.

Die Auslastung des Gesamtsystems ließ sich auch durch geschickte Kombination von Konsument_innen mit komplementärem Verbrauch verbessern. Neben der Beleuchtung begannen Zentralstationen zunehmend auch kleine Gewerbe, Industrie und Straßenbahnen mit Antriebskraft zu versorgen. Die Kraftauslastung durch Gewerbe und Verkehr fand vor allem während des Arbeitstages statt und komplementierte damit das Muster der Privathaushalte, die Beleuchtung vornehmlich morgens und abends nutzten. Jede_r städtische Nutzer_in wies eine charakteristische Verbrauchskurve auf, die in der Kombination die typische Verbrauchskurve einer Klein- oder Großstadt ergab (Abb. 7/8). Samuel Insull formalisierte die Verschiedenheit der Verbraucher_innen in einem <diversity factor>, der die Abweichung der maximalen Auslastung des Systems von der Summe aller einzelnen Maxima bezeichnete. Je weniger Anteil ein_e Verbraucher_in an der höchsten Auslastung des Systems hatte - wie die Zementfabrik und der_die Eisverkäufer_in, die im Winter den Betrieb einstellen -, desto wertvoller war er_sie für das System (Abb. 9).

Der Betreiber eines Elektrizitätswerkes kontrollierte einen Fluss potenzieller Arbeitskraft, der nicht gespeichert werden konnte. Dieser Fluss musste technisch <am Laufen〉 gehalten, vor allem aber wirtschaftlich aktualisiert, d.h. verwertet werden. Dem entsprach ein Blick auf die Stadt, der sie nach ihrer Möglichkeit vermaß, elektrische Arbeit zu absorbieren. Im Medium des Stroms über Zeit - dem Belastungsprofil - gewann die Vielfalt städtischer Industrien eine Form, die vom System gelesen und genutzt werden konnte. Der Verbrauch einer_s Nutzer_in schloss dabei den einer_s anderen nicht aus, sondern konnte ihn auch ermöglichen: «[W] hat cannot be produced economically separately can be produced economically as a whole. $>^{31}$ Belastungsprofile waren jedoch weit mehr als eine betriebsinterne Dokumentation: Die elektrotechnische Industrie 
war durch Patente, Lizenzen und Investitionen international so verflochten, dass Informationen über Auslastung und Betriebsmanagement weit zirkulieren und zur Planung neuer Anlagen genutzt wurden. In diesem elektrotechnischen Diskurs entstanden charakteristische Belastungskurven, die keiner_m einzelnen Nutzer_in mehr entsprachen, sondern von der Ähnlichkeit städtischer Strukturen im frühen 20. Jahrhundert ausgingen: $\mathrm{Ob}$ in Berlin, St. Petersburg oder Buenos Aires, die Straßenbahn fährt von frühmorgens bis spätabends.

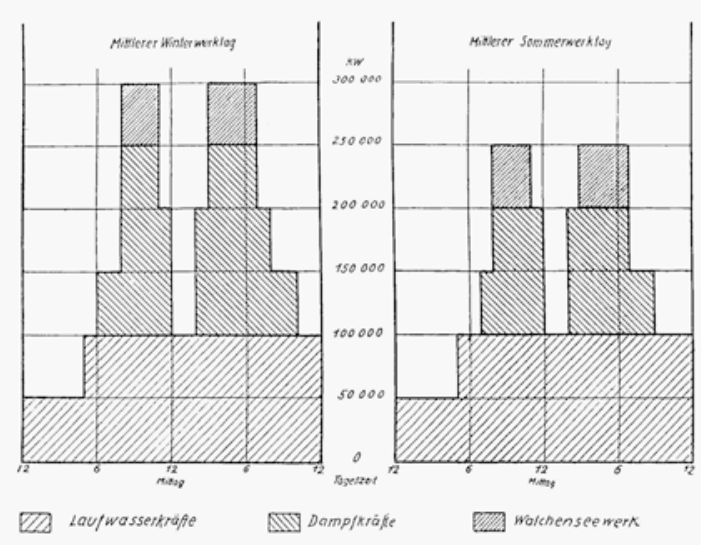

\section{Die <nationale Kraftwirtschaft >}

Der Erste Weltkrieg beschleunigte die Entwicklung, in der ehemals städtische Systeme zu regionalen und nationalen zusammengelegt wurden: Elektrische Anlagen wurden verstaatlicht, ausländische Investoren enteignet und Industriebetriebe gezwungen, ihre Elektrizität in ein allgemeines Netz einzuspeisen, von dem aus sie je nach Kriegsrelevanz verteilt werden konnte. ${ }^{32}$ Der Abbruch globaler Ressourcenströme lenkte den Blick auf eine intensivere Entwicklung des Staatsgebietes, und die Elektrizität erlaubte die Verwertung bisher ungenutzter <einheimischer> Ressourcen. Große elektrische Systeme symbolisierten die in der Zwischenkriegszeit vorherrschende Idee einer rational geplanten Wirtschaft besonders eindrücklich. ${ }^{33}$ Vor diesem Hintergrund könnte man - nach einer wissenschaftlichen und wirtschaftlichen - von einer spezifisch politischen Aneignung der Elektrizität sprechen, in der Elektrizitätssysteme zu einem Medium volkswirtschaftlicher Entwicklung wurden.

Das nationale Verbundsystem ist der deutlichste Ausdruck dieser politischen Aneignung der Elektrizität. Verbundsysteme hatten sich schon in privaten Unternehmen entwickelt, ${ }^{34}$ benötigten zu ihrer Vergrößerung in der Regel jedoch politische Unterstützung. Ein Verbundsystem umfasste nicht nur eine Vielfalt von Verbraucher_innen, sondern auch eine Vielfalt von Erzeuger_innen. Es komplementierte Wasserkraftwerke mit thermischen Kraftwerken, um mehrere Städte und Industrien zu versorgen (Abb. Io). ${ }^{35}$ Im Medium der Belastungskurve tauchen nun nicht nur die Bevölkerung, Gewerbe und Industrien, sondern auch die staatlichen Kohle- und Wasserressourcen auf. Verbundsysteme versprechen, die soziale, wirtschaftliche und geografische Vielfalt moderner Gesellschaften in einer Einheit aufzuheben: Der_Die Arbeiter_in konnte günstig und effizient mit Strom versorgt werden, nicht obwohl, sondern weil es die moderne Papierfabrik gab. So ermöglichten die Verbundsysteme ein «seamless web: the unity of land and water and men». Diese Utopie einer technologischen Versöhnung von Stadt und Land, Arbeit und Kapital, Natur und Kultur sprach aus vielen Elektrifizierungsprojekten der Zwischenkriegszeit. ${ }^{36}$

Abb. 10 Schematische Leistungskurve des Bayernwerks, eines Verbundsystems mit Wasser- und Dampfkraft, 1926
33 Vgl. Walther Rathenau: Die neue Wirtschaft, Berlin 1918; Carl Ballod: Der Zukunftsstaat. Produktion und Konsum im Sozialstaat, Stuttgart 1919; Frederick Winslow Taylor: The Principles of Scientific Management, New York 1913.

34 Hughes: Networks of Power, 419-428.

35 World Power Conference: Transactions of the World Power Conference, Sectional Meeting, Basel 1926, Bd. II, Section C: "The Economic Relation between Electrical Energy produced hydraulically and Electrical Energy produced thermally", Basel 1927.

36 David E. Lilienthal: TVA. Democracy on the March, New York 1944, 58; Gleb M. Krzhizhanovskii: Zadachi Energeticheskogo Khosyaystva, in: Planovoe Khosyaystvo, Bd. 6, 1928, 7-43. 


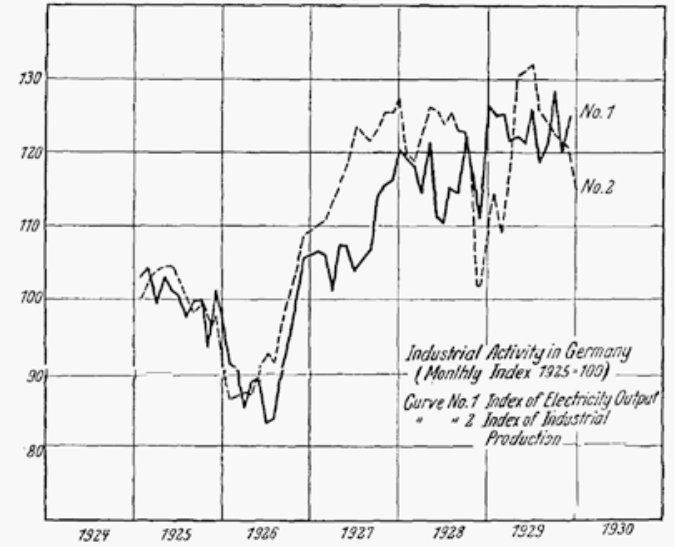

Erst die Elektrifizierungspolitik maximierte die Stromnutzung um jeden Preis. Es ging nicht um die Deckung, sondern um die Entwicklung eines Verbrauchs ${ }^{37}$ - um die Durchdringung der Volkswirtschaft mit Elektrizität. ${ }^{38}$ Die elektrotechnische Industrie hatte freilich immer schon die Stromnutzung motiviert: Zu diesem Zweck wurden elektrotechnische Geräte vermietet und Preise gesenkt. Diese Entwicklungstendenz fand jedoch ihre betriebswirtschaftliche oder kartellrechtliche Grenze. ${ }^{39}$ Nun sollten auch die Landwirtschaft und weniger wohlhabende Haushalte elektrifiziert und mit Elektrogeräten aller Art versorgt werden. Nicht nur die Höhe der Stromproduktion, sondern auch des Ver-

Abb. 11 Elektrizitätsproduktion als volkswirtschaftlicher Index im Vergleich, 1930
37 Leslie Hannah: Electricity before Nationalisation. A Study of the Development of the Electricity Supply Industry in Britain to 1948, London 1979, 150-161.

38 Hugh Quigley: Electrical Power and National Progress, London 1925.

39 Zum Kartellverfahren gegen Samuel Insull siehe Jeremiah D. Lambert: The Power Brokers: The Struggle to Shape and Control the Electric Power Industry, Cambridge 2015, Kap. 1; Hughes: Networks of Power; zur politischen Regulierung generell siehe Coopersmith: When Worlds Collide.

40 Hugh Quigley: Electricity as an Index of Industrial Production and Employment, in: The Transactions of the Second World Power Conference, Bd. XVI: World Problems of Power Economics, Berlin 1930, 95-127; Robert Sibley: America's answer to the Russian challenge, in which electric power, as a common denominator, is requisitioned to throw light on the Russian enigma and the challenge it presents to western civilization, San Francisco 1931.

41 Maria Falkner-Smit: The Motor Power Outfit of Labour and its Economic Efficiency, in: The Transactions of the Second World Power Conference, Bd. XVI: World Problems of Power Economics, Berlin 1930, 60-71. brauchs wurde nun Ausweis volkswirtschaftlicher Entwicklung.

Je mehr Tätigkeiten sich in der Stromnutzung dokumentierten, desto besser ließ sich der Strom durch die Volkswirtschaft und die Volkswirtschaft durch den Strom studieren. ${ }^{40}$ Die Konjunkturforschung ging ein in die Planung von Elektrizitätssystemen und die Elektrizitätsproduktion wurde als Index wirtschaftlicher Aktivität gehandelt (Abb. I I). Doch Strom ist kein neutrales Medium, das durch die Wirtschaft fließt, sie aufzeichnet und dabei unangetastet lässt. Strom ist eine wertbildende und schöpferische Kraft - Arbeitskraft. Die Elektrifizierung wurde Gegenstand der Produktivitätsforschung, die ihren Einfluss auf Wirtschaftszweige maß, um die volkswirtschaftlichen Orte zu lokalisieren, an denen das zirkulierende Arbeitspotenzial sich am effektivsten verwirklichte. ${ }^{41}$

In den Händen von Staaten wurde die Logik der Entwicklung von Elektrizitätssystemen entgrenzt, indem sie als Frage volkswirtschaftlicher Entwicklung und geopolitischer Macht reformuliert wurde..$^{42}$ Das Elektrizitätssystem wurde von einer betriebs- zu einer volkswirtschaftlichen Informationstechnologie, die die Durchdringung der Wirtschaft mit Energie aufzeichnet. In der politischen Aneignung wurde Strom zu einem Fluss künstlicher Arbeitskraft, der sich volkswirtschaftlich verwerten musste, indem er die allgemeine Produktivität steigerte. Die Elektrifizierung ist der Hebel, mit dem eine gegebene Bevölkerungsgröße und Ressourcenausstattung in ein Vielfaches an Wirtschaftsleistung umgesetzt werden konnte. ${ }^{43}$

\section{Energiewirtschaft $\mathbf{1 8 8 0 / 1 9 3 0}$}

Während Menschen, Tiere und Dampfmaschinen im I9. Jahrhundert noch verschiedene Ausdrucksformen eines produktiven Universums sind, ist der Mensch kein Teil der Kraftwirtschaft mehr. Wo er noch in den Kraftstatistiken auftaucht, geht es darum, den Wandel hin zu einer Wirtschaft auf Grundlage technisch kontrollierter Energieflüsse zu dokumentieren. Die Kraftwirtschaft schließt 
vieles ein, was im I9. Jahrhundert nicht Teil der Energiewirtschaft war, weil es nicht die Form der Antriebskraft hatte: Beleuchtung, Wärme, vielfältige Gewerbe- und Haushaltsaktivitäten. Was immer an das zirkulierende Arbeitspotenzial angeschlossen ist, kann durch sein Belastungsprofil studiert werden.

In den ersten Jahrzehnten des 20. Jahrhunderts entscheidet sich, wer am Fluss dieser künstlichen Arbeitskraft teilhat, wessen Nutzung gemessen und analysiert, angeregt oder gebremst wird. Für eine Technikgeschichte der Energie bliebe dies die Frage, wer mit Elektrizität versorgt werden kann; eine Geschichte der Aufschreibesysteme kann zeigen, wie das energiewirtschaftlich Plausible und Machbare sich über die dokumentierten und verarbeiteten Informationen bestimmt. Sofern sie zu einem eigenständigen Untersuchungsobjekt wird, hat die Formierung der Kraftwirtschaft auch Folgen für die Einschätzung, wie schnell ein Elektrizitätssystem wachsen kann und sollte, wie der Tarif die Nutzung beeinflusst, wie sich Kohleverbrauch zu Elektrizitätsnutzung verhält oder wie Elektrifizierung und wirtschaftlicher Wohlstand zusammenhängen. Das Experimentieren mit Elektrizitätssystemen der ersten Hälfte des 20. Jahrhunderts hat Folgen für die Energieentwicklungspolitik der zweiten Hälfte. ${ }^{44}$

Um I930 entstehen die ersten Statistiken, die alle volkswirtschaftlichen Energieflüsse dokumentieren sollen. Energiebilanzen erweitern den kraftwirtschaftlichen zu einem energiewirtschaftlichen Diskurs, insofern sie die wirtschaftlich relevante Energie mit der energetisch relevanten Wirtschaft identifizieren. Zu der Zeit ist durchaus noch gegenwärtig, dass man Menschen, Tiere, aber auch jegliche nicht-kommerzielle Energie aus der Energiewirtschaft verbannt hatte. Aus diesem Grund, so Oscar C. Miller von der US Federal Power Commission, sei diese Art der Buchhaltung auch «fundamentally deceptive: it was not possible to get a real $\langle$ Balance of Energy $\rangle$. There were tremendous sources of energy which could not be included.» ${ }^{45}$ Dennoch setzen sich Bilanzen als Informationsmedium einer entstehenden Energiepolitik in der zweiten Hälfte des 20. Jahrhunderts durch. Da außerhalb der Kraftwirtschaft die Dokumentation der Konsumption nicht mit der technischen Herstellung einhergeht, ist die Logik dieses neuen Aufschreibesystems eine bürokratisch-statistische: Die Produktionsdaten müssen zentral ermittelt, der Konsum muss geschätzt werden. Im Zuge der Umwelt- und Konservierungspolitik der I 97oer Jahre kann ein solcher von der Energieherstellung getrennter statistischer Apparat schließlich sogar die nicht-kommerzielle Energie aufzeichnen und wirtschaftlichen sowie entwicklungspolitischen Unternehmungen zugänglich machen.
42 In einer Welt, die vermessen und verteilt war, stimmten Imperialist_innen und Anti-Imperialist_innen darin überein, dass sich politische Macht nicht mehr in räumlicher Expansion, sondern in wirtschaftlicher Stärke zeige, in "the struggle for relative efficiency". Siehe dazu H. J. Mackinder: The Geographical Pivot of History, in: The Geographical Journal, Bd. 23, Nr. 4, 1904, 421-437, hier 422; siehe auch Gleb M. Krzhizhanovskii: Perspektivy elektrifikaciye, in: Planovoe Khozyaystuo, Bd. 2, 1925, 3-21, hier 12. Elektrifizierung gab dieser neuen, sintensiven, Wirtschaftspolitik eine konkrete Form.

43 Nirgends wird das deutlicher als in dem Entwicklungsmaß, das sich die junge Sowjetwirtschaft in ihrem ersten Fünfjahresplan gibt: dem Grad der «Energieausstattung lebendiger Arbeit», dem Verhältnis von technisch hergestellter zu menschlicher Arbeit, Gosplan: Peruyi Pyatiletnii Plan NarodnoKhozyaystvennogo Stroitel'stua SSSR, Moskau 1930, 23.

44 David Ekbladh: Meeting the Challenge from Totalitarianism. The Tennessee Valley Authority as a Global Model for Liberal Development, 1933-1945, in: The International History Review, Bd. 32, Nr. 1, 2010, 47-67; Vincent Lagendijk: Streams of knowledge. River development knowledge and the TVA on the river Mekong, in: History and Technology, Bd. 35, Nr. 3, 2019, 316-337.

45 World Power Conference: Minutes of the Meeting of the International Executive Council (Den Haag, 17.7.1935), 24, einsehbar im World Energy Council, London. 